\title{
Anesthetic experiences of severe mitral regurgitation and atrial septal defect newly appeared during the operation after myxoma removal surgery
}

\author{
Kwangrae Cho, Sang-Min Sin, Myoung-Hun Kim, and Wonjin Lee \\ Department of Anesthesiology and Pain Medicine, Busan Paik Hospital, College of Medicine, Inje University, Busan, Korea
}

Cardiac myxomas are the most common primary cardiac tumors, accounting for approximately $50 \%$ of all such tumors [1-3]. Around $75 \%$ of cardiac myxomas are detected in the left atrium (LA), and $15-20 \%$ in the right atrium (RA). Most myxomas arise from the interatrial septum at the fossa ovalis, but in some cases, myxomas have been reported to be attached to valve leaflets or in other locations in the atrium or ventricle [2,3]. The clinical signs and symptoms vary based on location, size, and mobility of myxoma. Most patients are symptomatic, and the triad of symptoms consists of embolism, intracardiac obstruction, and constitutional manifestations. Considering that cerebral and systemic embolism and even sudden death can be caused by cardiac myxomas, surgery is urgently need $[1,3]$.

A 61-year old woman without any medical history, she complained palpitations that started 20-days prior to her visit. On examination, her heart rate was regular, and her breath sounds were clear with no audible cardiac murmur. The patient denied having any symptoms that suggested a peripheral, cerebral, systemic, or coronary arterial embolism. A transthoracic echocardiogram (TTE) showed a pedunculated circular mass attached to the interatrial septum within the LA measuring $27.87 \mathrm{~mm} \times$ $20.14 \mathrm{~mm}$. It also showed a normal sinus rhythm with normal ventricular wall thickness and contraction. The LA was enlarged, and the mitral valve (MV) leaflets were mildly thickened and calcified with no regurgitation. From the TTE, clinical impression was cardiac myxoma.

On arrival in the operating room, electrocardiogram, pulse oxymeter, and non-invasive blood pressure were monitored, and a left radial arterial line was placed before anesthetic induction. Anesthetic induction was begun with $15 \mathrm{mg}$ of ectomidate, 30 $\mu \mathrm{g} / \mathrm{kg}$ of fentanyl, and $0.2 \mathrm{mg} / \mathrm{kg}$ of vecuronium, and anesthesia was maintained with $10 \mu \mathrm{g} / \mathrm{kg} / \mathrm{hr}$ of fentanyl, $50 \mu \mathrm{g} / \mathrm{kg} / \mathrm{hr}$ of midazolam, and $0.2 \mathrm{mg} / \mathrm{kg} / \mathrm{hr}$ of vecuronium. During the operation, a transesophageal echocardiogram (TEE) was performed to confirm the presence of cardiac mass and to detect changes in ventricular wall motion and cardiac valves.

The echocardiographic appearance of the mass was typical for atrial myxoma. In mid-esophageal short axis view, a $1.80 \mathrm{~cm}$ $\times 3.63 \mathrm{~cm}$ sized circular mass from the interatrial septum was observed (Fig. 1A). In mid-esophageal four chamber and long axis views, a left atrial mass was observed with no MV regurgitation. In the TEE, no other cardiac problems were detected.

Arterial cannulation was done in upper ascending aorta, and bicaval venous cannulation was done in superior vena cava and lower RA. Cardiopulmonary bypass (CPB) then followed, with an aortic cross clamp and an infusion of cold cardioplegia via aortic root cannula. Right atrial wall and interatrial septum were incised, and left atrial mass including left atrial dome was removed. The defects associated with left atrial dome, and the incision in the interatrial septum and right atrial wall were closed with sutures using prolene. After release of aortic cross clamp and direct current cardioversion, the heart beat recovered. A repeat TEE showed the removal of left atrial mass and an intact interatrial septum with no shunting; however, severe MR was

Corresponding author: Kwangrae Cho, M.D., Department of Anesthesiology and Pain Medicine, Busan Paik Hospital, College of Medicine, Inje University, Gaegeum-dong, Jin-gu, Busan 614-735, Korea. Tel: 82-51-890-6520, Fax: 82-51-898-4216, E-mail: maeteost@hanmail.net (c) This is an open-access article distributed under the terms of the Creative Commons Attribution Non-Commercial License (http:// creativecommons.org/licenses/by-nc/3.0/), which permits unrestricted non-commercial use, distribution, and reproduction in any medium, provided the original work is properly cited. 

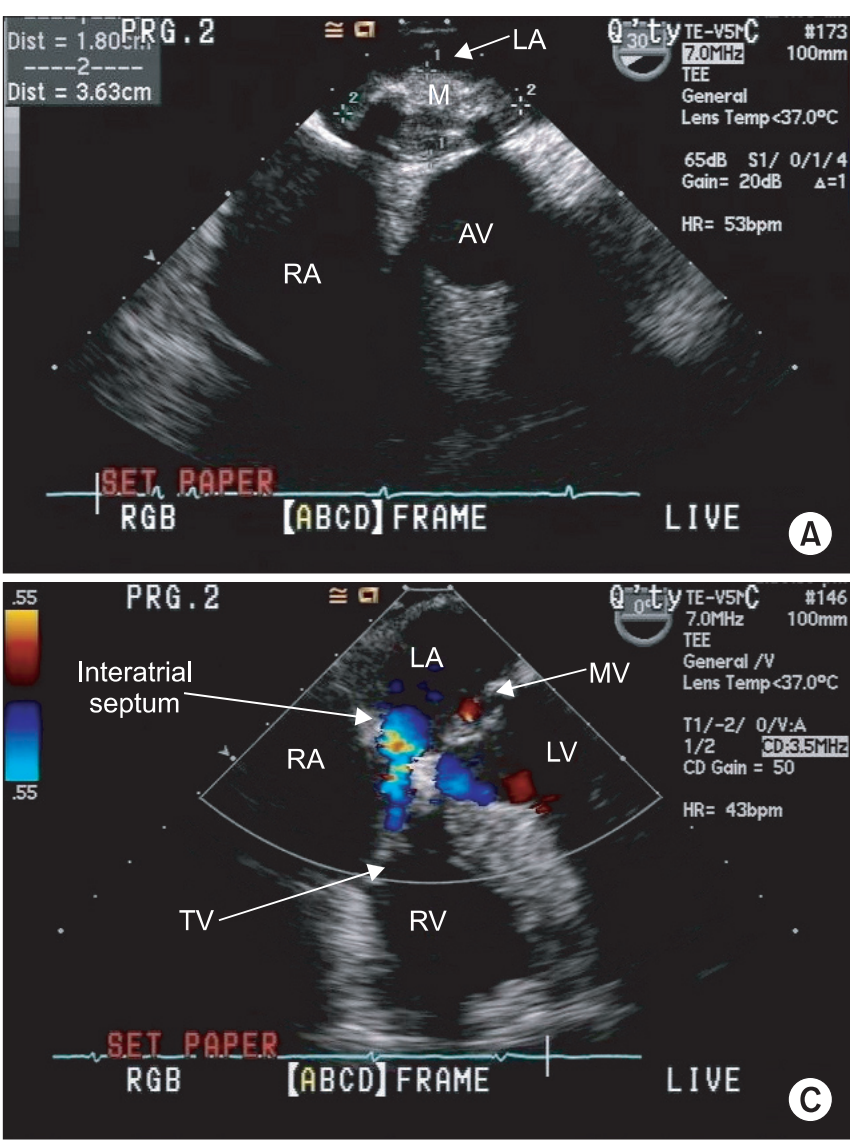

newly observed. On Doppler color flow image, the amount of regurgitation jet was over 50\% in LA, and the spectral Doppler was dense (Fig. 1B). After an urgent consultation with surgeon, the operation was restarted to correct the MV regurgitation.

Through an incision in the LA and RA, the MV was exposed and explored. The leaflets were relatively good, but the mitral annulus was distorted due to a nearby suture in the left atrial dome. To repair the mitral annulus, a St. Jude Medical Tailor ${ }^{\circledR}$ annuloplasty ring $27 \mathrm{~mm}$ was inserted.

After mitral annuloplasty, a repeat TEE showed only trivial MR. However, left to right jet flow was detected in the interatrial septum by the color Doppler in the mid-esophageal four chamber view (Fig. 1C). The operator supposed that interatrial septal defect was caused by the procedure to repair the MV. It was closed with a bovine pericardial patch.

The patient was weaned from the CPB, and her vital signs were stable without any medication. In addition, the TEE showed only trivial MR and an intact interatrial septum with no shunting. No residual cardiac mass was detected.

The operation lasted 10 hours and 50 minutes, and the $\mathrm{CPB}$ lasted 6 hours and 25 minutes. After the operation, the patient was transferred to the intensive care unit and treated with mechanical ventilator under sedation. The day after the surgery, the

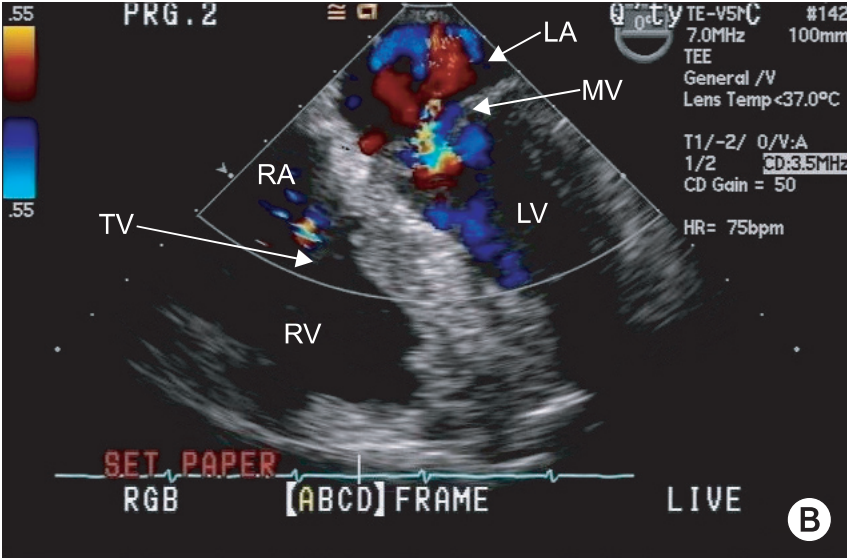

Fig. 1. (A) Intraoperative transesophageal echocardiography, midesophageal short axis view, a $1.80 \mathrm{~cm} \times 3.63 \mathrm{~cm}$ sized circular mass from the interatrial septum was observed with slightly enlarged left atrium. (B) Mid-esophageal four chamber view, the left atrial mass was removed, and severe mitral regurgitation was newly observed. (C) Mid-esophageal four chamber view, this figure showed trivial mitral regurgitation, and a defect of interatrial septum was detected and left to right jet flow was detected in the interatrial septum by the color Doppler. LA: left atrium, $\mathrm{RA}$ : right atrium, $\mathrm{LV}$ : left ventricle, $\mathrm{RV}$ : right ventricle, $\mathrm{AV}$ : aortic valve, MV: mitral valve, TV: tricuspid valve, M: mass.

patient became alert and was weaned off the ventilator. The patient stabilized, 1 week later, and was transferred to the general ward. She then had an uneventful recovery and was discharged from the hospital 24 days after the surgery.

Atrial myxoma may cause MV obstruction or mitral insufficiency $[4,5]$. If the tumor is large, soft, easily deformable, and mobile, the potential for the occurrence of a MV abnormality is high. As atrial myxoma moves back and forth between the atrium and ventricle, it causes damage to atrioventricular valve resulting in valve insufficiency or valve obstruction; this mechanism is called the "wrecking ball" effect. In addition, the mechanical pressure of tumor causes dilatation of valve annulus and deformation of the valve [4]. Considering other case reports in which MV regurgitation was newly detected during the operation, it seems probable that MR was masked by myxoma [4,5].

Although the echocardiogram must be examined to study cardiac myxomas before the operation, sometimes the presence of MV pathology cannot be detected. Using intraoperative TEE, we can confirm the location and size of myxoma as well as other valve pathology not detected before the operation. In addition, it is useful to estimate the residual cardiac mass and cardiac function and to evaluate heart valve after removal of myxoma.

In conclusion, surgical removal of a large myxoma can pro- 
duce postoperative configurational changes in adjacent cardiac structures including the MV annulus, resulting in postoperative MV insufficiency. Therefore, the importance of intraoperative
TEE cannot be overemphasized in evaluating myxomas as well as possible associated postoperative structural changes in adjacent cardiac structures.

\section{References}

1. Yuan SM. Mitral valve myxoma: clinical features, current diagnostic approaches, and surgical management. Cardiol J 2012; 19: 105-9.

2. Matsushita T, Huynh AT, Singh T, Hayes P, Armarego S, Seah PW. Mitral valve annular dilatation caused by left atrial myxoma. Heart Lung Circ 2009; 18: 145-7.

3. Oliveira R, Branco L, Galrinho A, Abreu A, Abreu J, Fiarresga A, et al. Cardiac myxoma: a 13-year experience in echocardiographic diagnosis. Rev Port Cardiol 2010; 29: 1087-100.

4. Fujii A, Inaoka M. Huge left atrial myxoma masking the severity of mitral regurgitation; report of a case. Kyobu Geka 2008; 61: 410-3.

5. Formica F, Sangalli F, Paolini G. Unusually large left atrial myxoma causing mitral valve occlusion and hiding a severe mitral regurgitation: a case report. Heart Surg Forum 2006; 9: E849-50. 